\title{
Pushing lattice QCD to the edge: flavor physics
}

\author{
Alessandro Cerri ${ }^{1}$ \\ Lawrence Berkeley National Laboratory \\ 1, Cyclotron Rd Berkeley 94720 CA., USA \\ E-mail: alessandro.cerri@gmail.com
}

While CKM angles often exhibit clean relationships with standard model observables, the sides of the unitarity triangle often require lattice computations in order to extract fundamental Standard Model parameters from experimental data. I will cover some of the most recent advances in flavor physics, in particular in the $\mathrm{b}$ and $\mathrm{c}$ sectors. While the former sees lattice QCD as one of the most important (if not the most important) bottlenecks towards the clean extraction of CKM parameters, the latter provides an environment with better determined CKM parameters, where experimental results can critically probe LQCD.

XXIV International Symposium on Lattice Field Theory

Tucson, Arizona US

23-28 July, 2006

\footnotetext{
1 Speaker
} 


\section{Introduction}

Lattice computations have become an extremely valuable tool in the framework of heavy flavor physics. The interaction between the two fields is in fact to mutual advantage: while on one hand flavor physics allows - with its measurements - the testing of lattice techniques on the problems of interest, on the other it benefits from the precision with which lattice connects phenomenological parameters to the underlying fundamental standard model quantities.

This paper will provide an overview of experimental results available at the time of the Lattice 2006 conference. Far from being exhaustive and up-to date, this overview will focus on stigmatizing the above mentioned interchange between the two fields.

\subsection{Scope}

It is almost unavoidable to follow the author's field of activity and taste for experimental challenges in this kind of reviews, usually partial with respect to experiments, coverage of results, and time window. This proceeding will not be an exception: the topics covered will be experimental results in the fields of $b$ and $c$ physics, following an arbitrary selection of those which serve better the purpose of illustrating my argument.
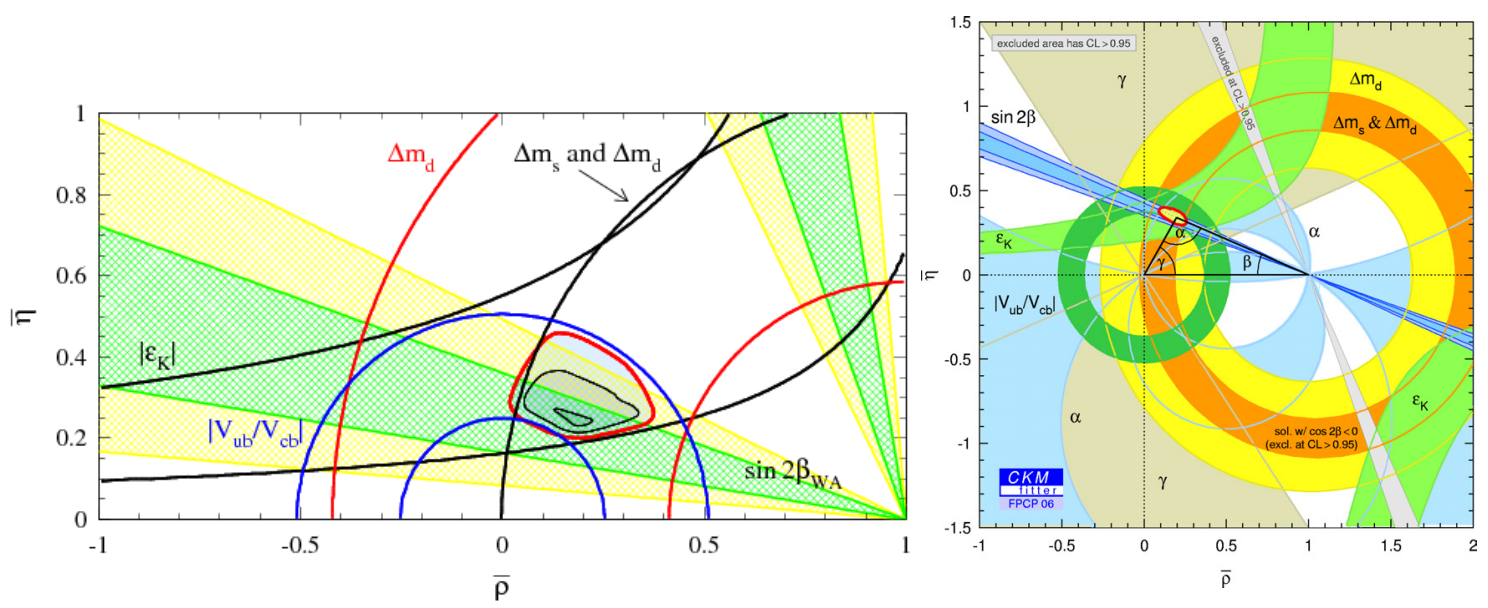

Figure 1: evolution of the determination of the vertex of the unitarity triangle between 2001 (left) and 2006 (right) as obtained by [1]. The various lines and bands represent constraints from different experimental measurement. The red closed line indicates the $90 \% \mathrm{CL}$ band in the left plot, and the $95 \%$ CL band in the one on the right.

\subsection{Why focus on flavor physics?}

Figure 1 compares two snapshots of the constraints on the knowledge of the position of the vertex of the unitarity triangle: the status in 2001 (when the knowledge of the B meson phenomenology was on the verge of exploding) and that of spring 2006. The two pictures are very different thanks to the experimental determination of several quantities, connected to the measurement of angles and sides of this triangle. 
The measurement of angles of the Cabibbo-Kobayashi-Maskawa (CKM) triangle presents challenges, but often proves to be rather straightforward in the connection between experiment and the standard model. On the other hand, the determination of the sides of the triangle tends to be limited by the uncertainty with which the experimental numbers are connected to the length of those sides.

A striking example is the comparison between the information drawn from the measurement of the oscillation frequency of $\mathrm{B}_{\mathrm{d}}$ mesons $\left(\Delta \mathrm{m}_{\mathrm{d}}\right)$ and the measurement of the timedependent $\mathrm{CP}$ asymmetry in $\mathrm{B}_{\mathrm{d}} \rightarrow \mathrm{J} / \psi \mathrm{K}_{\mathrm{s}}$ decays, both of which have been compiled in PDG2006 [3]: the experimental quantities are measured with impressive precision - two of the many examples of how successful the $\mathrm{B}$ factories have been: $\Delta m_{d}$ is known to better than $1 \%$ and the $\mathrm{CP}$ violating amplitude in $\mathrm{B}_{\mathrm{d}} \rightarrow \mathrm{J} / \psi \mathrm{K}_{\mathrm{s}}$ is known at the level of 5\%. However, while the latter directly translates into a similar uncertainty on the standard model parameter $\sin (2 \beta)$, the former translates into a knowledge on the side of the CKM triangle opposed to the angle $\gamma$ which is at the level of $50 \%$ or so. Lattice QCD is actually the only instrument which allows to probe the non-perturbative QCD effects which affect the latter connection, and the uncertainty on its predictions directly affects our knowledge of the Standard Model.

\section{Experimental results}

This section will discuss several experimental results from different experiments, following an increasingly complicated connection between experimental quantities, lattice and or Standard Model.

I will begin covering fully leptonic decays of heavy flavored mesons, which benefit from a conceptually very simple modeling, making the connection between experiment, theory and LQCD extremely elegant. Semileptonic decays will then offer an increasingly complicated system where to probe both LQCD and the Standard Model, followed then by a discussion of the most recent results aiming at the determination of the sides of the CKM triangle, with particular attention to the importance of increasingly precise LQCD determinations.

\subsection{Fully leptonic decays}

When a charged B or D meson decays into a final state made uniquely of leptons, the process is very accurately described by the tree level annihilation of the $q \bar{q}$ pair into a $\mathrm{W}$ meson, which decays into a lepton and a neutrino. The branching fraction for such a process depends on the weak coupling constant, the relevant CKM coefficient, a phase space term and QCD corrections to the strong vertex in the $q \bar{q}$ annihilation:

$$
\frac{B(X \rightarrow l v)}{\tau_{X}}=\frac{G_{F}^{2} m_{X} m_{l}^{2}}{8 \pi}\left(1-\frac{m_{l}^{2}}{m_{X}^{2}}\right)^{2} f_{X}^{2}\left|V_{q_{X} \bar{q}_{X}}\right|^{2}
$$

This expression accurately describes the process. It also immediately explains several fundamental features of this type of decay, as well as showing that QCD enters into the process with one single coefficient: the decay constant $f_{X}$. 
This reveals a very immediate connection between lattice and the flavor sector: while on one hand a measurement of this branching ratio can be used to determine a CKM coefficient by plugging in a suitable LQCD determination of $f_{X}$, on the other it is possible to use the relationship and other experimental measurements of $V_{q \bar{q}}$ to cross-check $f_{X}$ predictions.

\subsubsection{Charmed mesons}

Performing the measurement of absolute branching fractions is greatly simplified in situations - like the CLEO-c case - where the meson is produced in pairs and on-threshold of a well defined resonance. This gives direct access to absolute branching fractions, and provides another essential advantage which is crucial for the reconstruction of fully leptonic decays: the knowledge that the event is composed exactly of a $D \bar{D}$ pair. In these conditions the momentum of the neutrino in the fully leptonic decay can be indirectly determined by "tagging" the decay via identification of one of the two $D$ mesons in a known observable decay (e.g. $D^{+} \rightarrow K \pi \pi$ ), and at the same time observing a muon in the detector. The neutrino energy can be indirectly obtained as well, using the knowledge of the energy of the incoming beams producing the dimeson resonance, and the energy of the muon itself. An example of this can be seen in the left side of Figure 2, where the square of the candidate neutrino mass is derived from its momentum and energy, showing - together with contributions from well identified reflections - a clear $\mathrm{D} \rightarrow \mu \nu$ peak.

In the context of fully leptonic decays of $D^{ \pm}$mesons, the relevant CKM coefficient is $V_{c d}$. Assuming $V_{c d}=V_{u s}$ and taking both $V_{u s}$ and the $\mathrm{D}^{+}$lifetime $\tau_{D^{+}}$from the 2004 world average of the Particle Data Group [1], the CLEO-c collaboration [4] measures $B\left(D^{+} \rightarrow \mu v\right)=\left(4.40+0.66_{-0.12}^{+0.09}\right) \times 10^{-4}$ and $f_{D^{+}}=\left(222.6 \pm 16.7_{-0.12}^{+2.8}\right) \mathrm{MeV}$. This sets the experiment and LQCD in the very interesting situation of comparable uncertainties, as illustrated by Figure 2 .
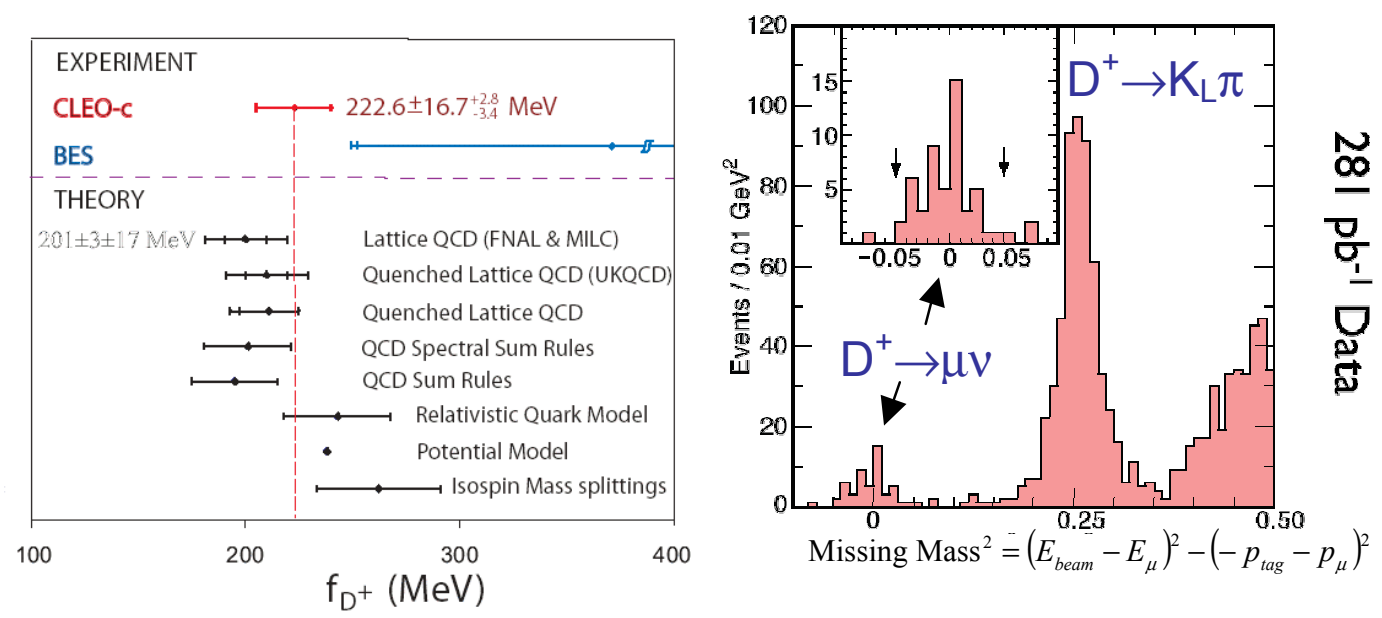

Figure 2: Left: comparison of the experimental measurements and lattice predictions for the $\mathrm{D}^{+}$decay constant. Right: missing mass distribution of the CLEO $\mathrm{D}^{+} \rightarrow \mu \nu$ candidates [4]. 
The BABAR and CLEO experiments recently measured [6] the analogous quantity for $\mathrm{D}_{\mathrm{s}}$ mesons. This gives both the possibility of comparing $f_{D_{s}}$ with the theoretical predictions, and a way of checking predictions for the ratio $f_{D_{s}} / f_{D^{+}}$, a sensitive check in view of analogous quantities useful in the determination of $\left|V_{t s} / V_{t d}\right|$. CLEO measures in fact $f_{D s}$ both in the $D_{s} \rightarrow \mu \nu$ and $D_{s} \rightarrow \tau \nu$ channels, which can be seen either as a check of lepton universality or a joint more precise measurement of $f_{D s}$.

\begin{tabular}{|lc|}
\hline$f_{D^{+}}$from LQCD (FNAL/MILC) & $201 \pm 3 \pm 17 \mathrm{MeV}$ \\
\hline$f_{D^{+}}$CLEO-c & $222.6 \pm 16.7 \pm \mathrm{MeV}$ \\
\hline$f_{D_{s}}$ BABAR & $279 \pm 17 \pm 6 \pm 19 \mathrm{MeV}$ \\
\hline$f_{D_{s}}$ CLEO $(\mathrm{D} \rightarrow \mu \nu$ only) & $282 \pm 16 \pm 7 \mathrm{MeV}$ \\
\hline$f_{D_{s}}$ CLEO $(\mathrm{D} \rightarrow \mu \nu$ and $\mathrm{D} \rightarrow \tau v)$ & $280.1 \pm 11.6 \pm 6 \mathrm{MeV}$ \\
\hline$f_{D_{s}} / f_{D^{+}}$from LQCD FNAL/MILC & $1.21 \pm 0.01 \pm 0.04 \mathrm{MeV}$ \\
\hline$f_{D_{s}} / f_{D^{+}}$BABAR+CLEO & $1.27 \pm 0.14 \mathrm{MeV}$ \\
\hline$f_{D_{s}} / f_{D^{+}}$CLEO $\left(\mathrm{D}_{\mathrm{s}} \rightarrow \mu \nu\right.$ and $\left.\mathrm{D}_{\mathrm{s}} \rightarrow \tau v\right)$ & $1.26 \pm 0.11 \pm 0.03 \mathrm{MeV}$ \\
\hline
\end{tabular}

Table 1: comparison of experimental [4] [6] (blue background) and LQCD [5] (yellow background) determination of the $\mathrm{D}^{+}$and $\mathrm{D}_{\mathrm{s}}$ decay constants, as well as their ratios.

Table 1 reports the experimental and lattice QCD determinations of the decay constants for $\mathrm{D}^{+}$and $\mathrm{D}_{\mathrm{s}}$ decays, as well as their ratios. The extremely good agreement among the different determinations proves how the knowledge of systematic uncertainties on the LQCD derivation of these numbers is well under control.

\subsection{2 $\quad B \rightarrow \tau v$}

$\mathrm{B}$ factories are in the same experimental situation with b-flavored mesons as CLEO-c is for c-flavored mesons: they produce di-meson pairs right on resonance, with the advantage of being able to obtain clean events and use fully reconstructed B on one side as "tags" for a search of signatures on the opposide ("signal") side. It is inevitable therefore that BABAR and BELLE would investigate fully leptonic decays of the $\mathrm{B}_{\mathrm{u}}$. The only publicly available information at the time of this paper is from the BELLE collaboration [7], looking for $\mathrm{B}_{u} \rightarrow \tau v$ decays ( $\tau$ is the most favored lepton in the final state because of the spin-disfavored configuration of the decay).

$B \bar{B}$ pairs are first identified in the detector with a fully reconstructed "tag" B - decaying in the $\mathrm{D}^{(*) 0}\left[\pi, \rho, \mathrm{a}_{1}, \mathrm{D}_{\mathrm{s}}{ }^{(*)}\right]$ modes - which yields 680000 tags with a purity of $55 \%$. The search proceeds with the reconstruction of $\tau$ candidates in the five different final states $(\mu \nu v, \mathrm{e} v, \pi \nu, \pi \pi \nu$, $\pi \pi \pi v$ ), accounting for about $80 \%$ of the $\tau$ width.

The distribution of additional energy deposited in the calorimeter after subtracting the contribution from all the known particles is then studied looking for a signal.

The whole procedure is first checked with $\mathrm{B} \rightarrow \mathrm{D}^{* 0} \mathrm{l} v$ decays on the "signal" side, and then applied to the $\tau v$ candidates (see Figure 3). 

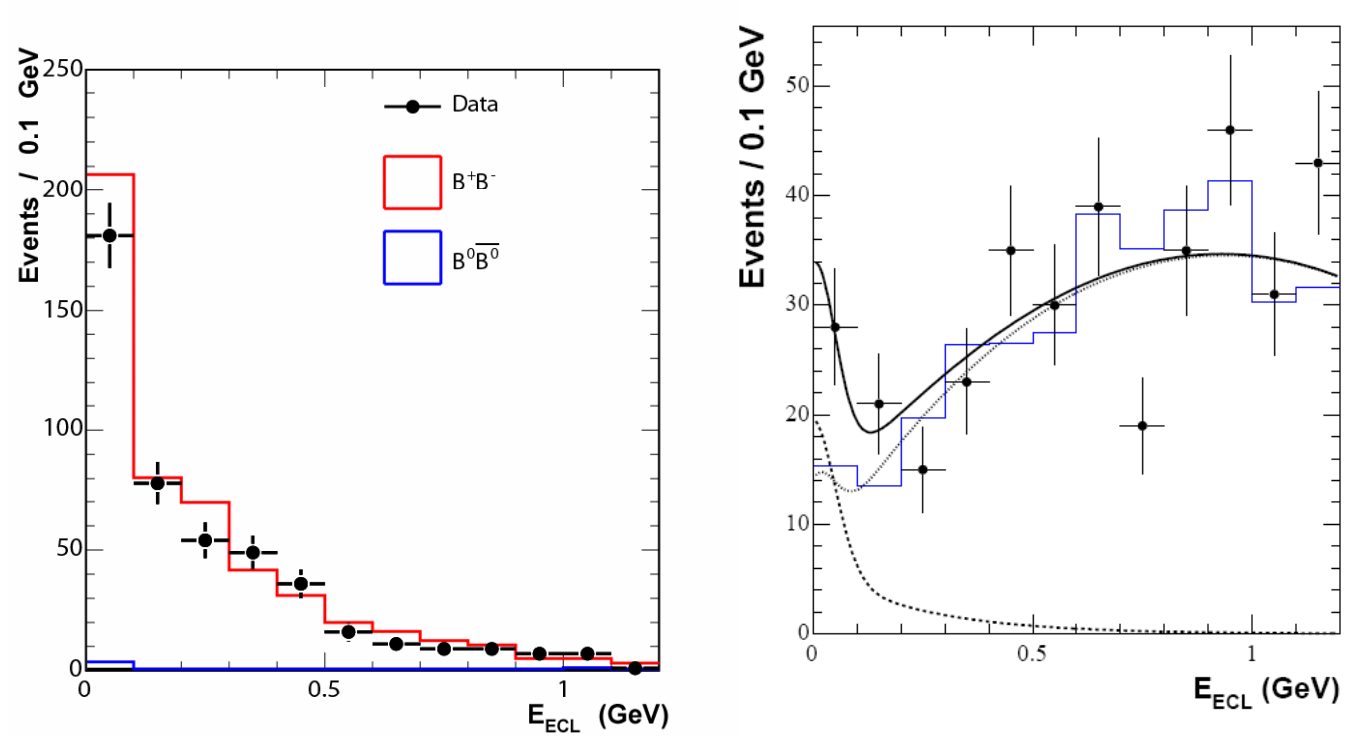

Figure 3: Left: distribution of missing energy for the control sample $\mathrm{B} \rightarrow \mathrm{D}^{* 0} l v$. The black data points are superimposed with the $\mathrm{B}^{+} \mathrm{B}^{-}$(red) and a small $\mathrm{B}^{0} \mathrm{~B}^{0}$-bar contribution predicted by montecarlo. Right: fit to the final distribution of missing energy for the $54 B_{u} \rightarrow \tau v$ candidates obtained from the five $\tau$ decay modes reconstructed in the analysis. The data points (black crosses) are superimposed to the final fit (continuous line) separated into the signal model (darker dots) and the background shape (lighter finely dotted line). The background functional form is modeled after the MC model obtained using random trigger data runs [7].

The fit returns $17.2_{-4.7}^{+5.3}$ signal events out of a total of 54 candidates, yielding a $3.5 \sigma$ significance once the preliminary studies of systematic sources are included. A measurement of the $\mathrm{B} \rightarrow \tau \nu$ branching fraction can be obtained in the signal hypothesis:

$$
B F\left(B^{+} \rightarrow \tau v\right)=\left(1.79_{-0.49-0.46}^{+0.56^{+0.39}}\right) \times 10^{-4}
$$

Using $\left|V_{u b}\right|=(4.39 \pm 0.33) \times 10^{-3} \mathrm{GeV}[8]$, BELLE derives the first direct measurement of $f_{B}=229_{-31}^{+36}-34 \mathrm{MeV}$, in good agreement with the more precise theoretical expectation of $f_{B}=216 \pm 22 \mathrm{MeV}$ obtained from LQCD [5].

\subsection{Semileptonic decays}

Semileptonic decays add one additional degree of complication to the picture, since the contribution from QCD corrections to the basic first order spectator diagram has now one additional degree of freedom. We can write for instance the differential partial width as a function of the lepton energy in the meson's rest frame in the case of a $\mathrm{D}_{\mathrm{s}}$ mesons:

$$
\frac{d \Gamma}{d q^{2}}=\frac{G_{F}^{2}}{24 \pi^{3}} p_{K}^{3}\left(q^{2}\right)\left|V_{c s}\right|^{2}\left|f_{+}\left(q^{2}\right)+O\left(m_{l}^{2}\right)\right|^{2}
$$


which allows in principle the extraction of $\left|V_{c s}\right|$ from a measurement of $\frac{d \Gamma}{d q^{2}}$. The problem is slightly more complicated by the fact that the phase space dependent term indicated by $p_{K}^{3}\left(q^{2}\right)$ is peaking around $q^{2}=0$, where LQCD computations, as well as experimental determinations, are most difficult.

A common experimental approach is - because of this - to revert to the determination of the shape of $\frac{d \Gamma}{d q^{2}}$ as a function of $q^{2}$, normalized to the value at $q^{2}=0:$ this is the case - for instance - of the BABAR [10] and FOCUS [11] experiments measuring $f\left(q^{2}\right)$ in semileptonic $\mathrm{D}^{0}$ decays with either kaons or pions. The BELLE collaboration measures instead the absolute shape of $\frac{d \Gamma}{d q^{2}}$ for $\mathrm{D}^{0}$ decays to , reported in [12]. Figure 4 shows these experimental results superimposed to the LQCD predictions from [5].
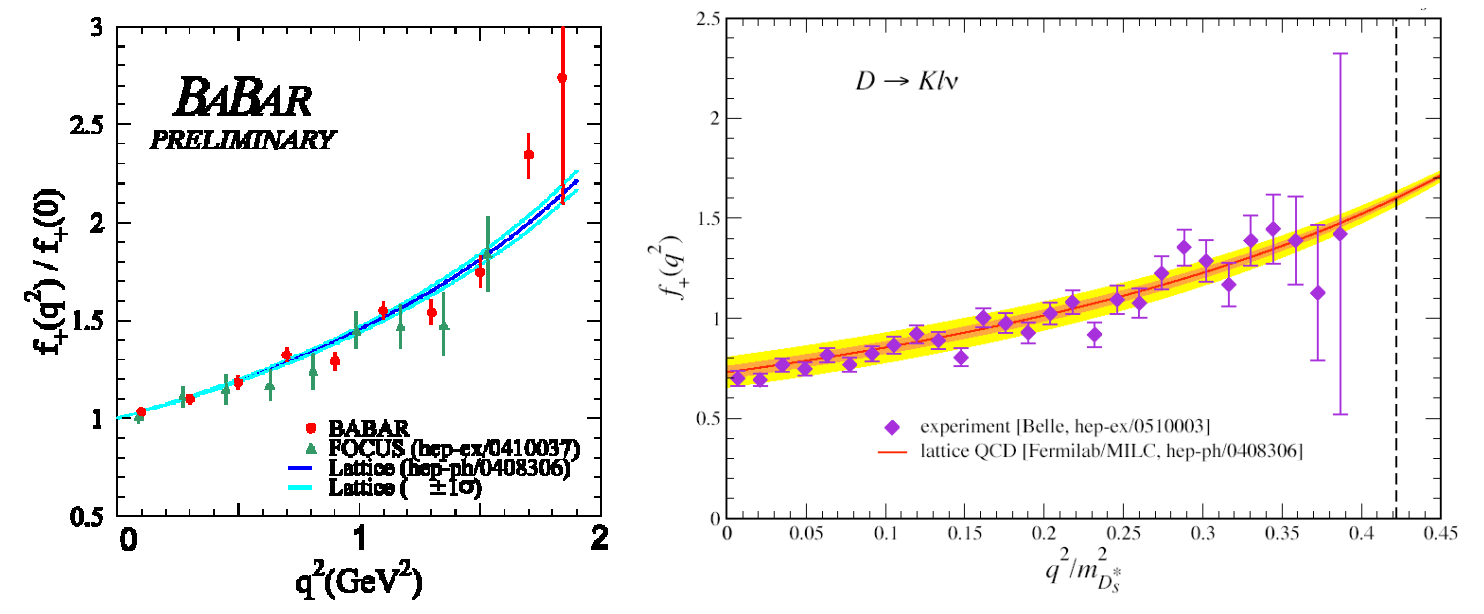

Figure 4: Left: comparison of the relative $\mathrm{q}^{2}$ dependence of the $\mathrm{D}^{0}$ form factor as determined by the BABAR [10] and FOCUS [11] collaborations with lattice predictions from [5]. Right: comparison of the absolute shape of $\frac{d \Gamma}{d q^{2}}$ for $\mathrm{D}^{0} \rightarrow \mathrm{Kl} v$ decays as determined by the BELLE collaboration [12], again compared to the lattice QCD predictions from [5].

Both methods yield a strikingly good agreement with the LQCD predictions from [5].

The CLEO experiment recently measured [13] the absolute scale and the shape of $f\left(q^{2}\right)$ for $D^{0} \rightarrow K l v$ and $D^{0} \rightarrow \pi l v$ decays - parameterized according to the phenomenological model: $\left|f_{+}\left(q^{2}\right)\right|=\frac{f_{+}(0)}{1-\frac{q^{2}}{m_{\text {pole }}^{2}}}$ [14]. Figure 5 compares this and all the other experimental results with the available LQCD predictions, showing remarkable agreement. 

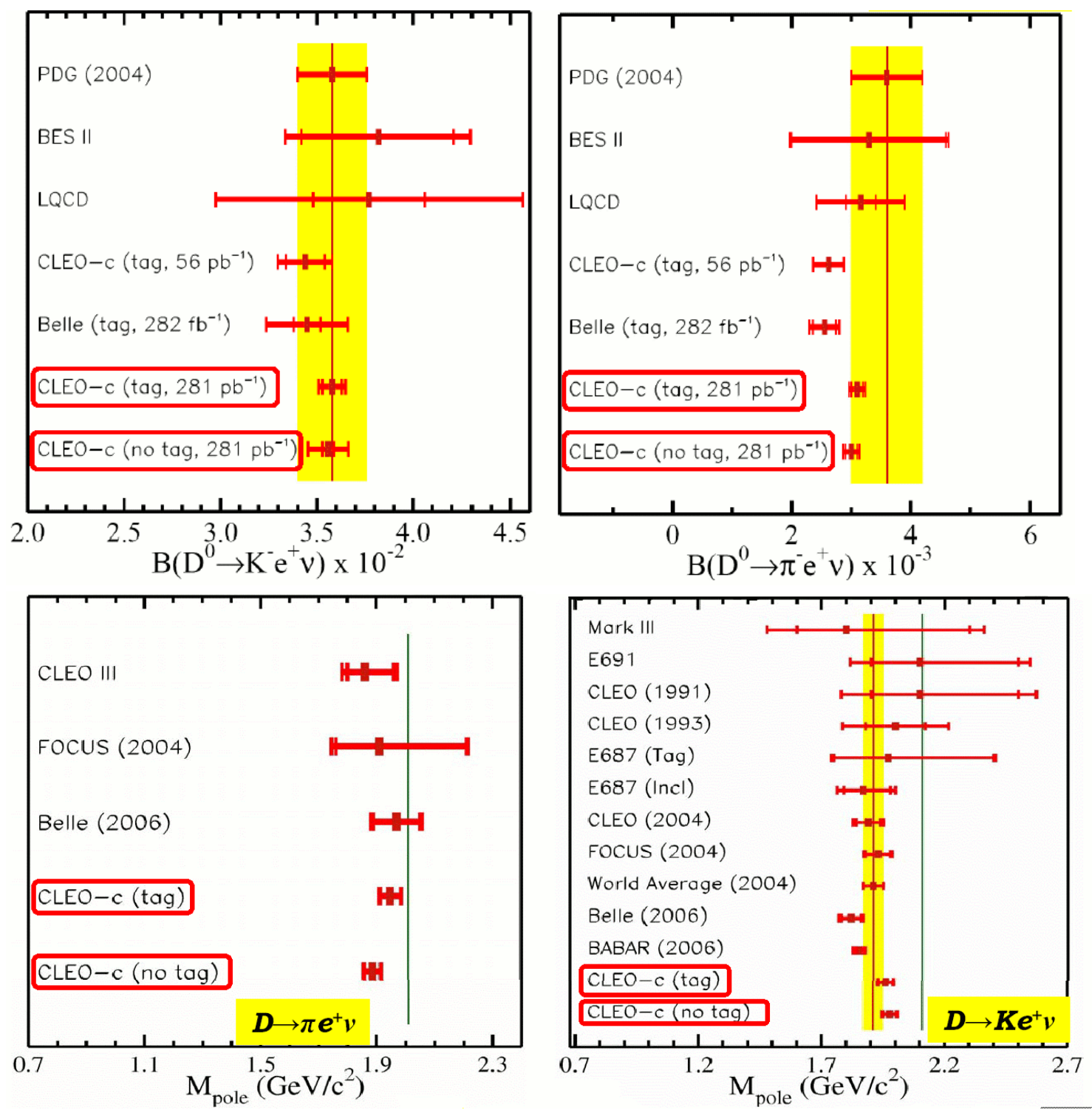

Figure 5: measurements of the absolute branching fraction (top) and the shape parameter $\mathrm{M}_{\text {pole }}$ (bottom) for $\mathrm{D} 0 \rightarrow \pi \mathrm{l} v$ (left) and $\mathrm{D} 0 \rightarrow \mathrm{Kl} v$ (right) decays from the various experiments reported in the text, compared to the latest LQCD predictions [5]. The yellow bands show - when available - the world average range for comparison [13].

While the studies carried on in the charm sector allow a check of the LQCD procedures, the transposition of the same approach to semileptonic B decays allows the most precise experimental determinations of the CKM coefficients $V_{u b}$ and $V_{c b}$. These determinations are to date [3] still significantly affected by systematic uncertainties in the connection between the experimental value of the branching fractions and the underlying CKM coefficients.

\section{$2.3 \quad \mathbf{V}_{\mathrm{td}} / \mathbf{V}_{\mathrm{ts}}$}

The determination of the side of the CKM triangle facing the angle $\gamma$ would proceed - in principle - through a measurement of $\left|V_{t d}\right|$. However this turns out not to be viable since the 
closest experimental quantities $\left(\Delta \mathrm{m}_{\mathrm{d}}\right.$ and the branching fraction for processes involving a $\mathrm{b} \rightarrow \mathrm{d} \gamma$ transition) are plagued by large QCD corrections that are difficult to keep under control. The only solutions to this problem experimentally explored so far rely on a normalization of the interesting process to a similar process involving an $s$ quark instead of a $d$ quark. For instance, instead of measuring the absolute oscillation frequency of $B_{d}$ mesons, one can try to factor out much of the hadronic uncertainties exploiting the ratio $\frac{\Delta m_{d}}{\Delta m_{s}}$, or analogously try to measure $\frac{\Gamma(b \rightarrow d \gamma)}{\Gamma(b \rightarrow s \gamma)}$. In the next two sections, the experimental situation for both approaches is briefly discussed, demonstrating another case where improvements in LQCD determinations would directly affect our knowledge of the Standard Model.

\subsection{1 $\quad \mathrm{B} \rightarrow \mathrm{s} \gamma$}

The BELLE experiment has measured in [14] both $b \rightarrow s \gamma$ and $b \rightarrow d \gamma$ transitions in the exclusive processes $B \rightarrow\left(\rho / \omega / K^{*}\right) \gamma$, where the multiple $b \rightarrow d \gamma$ decays are used to disentangle loop and annihilation diagram contributions.

The ratio of the combined $\mathrm{B} \rightarrow(\rho, \omega) \gamma$ branching fraction to the $\mathrm{B} \rightarrow \mathrm{K}^{*} \gamma$ one is evidently proportional to $\left|\frac{V_{t d}}{V_{t s}}\right|^{2}$ as well as the ratio of form factors in the two decays - which can be obtained through LQCD.

The resulting measurement is: $\left|\frac{V_{t d}}{V_{t s}}\right|=0.199_{-0.025}^{+0.026}(\exp )_{-0.015}^{+0.018}($ theo $)$, with an uncertainty still dominated by the experimental determination.

\subsection{2 $\quad$ B $_{\mathrm{s}}$ mixing}

The close similarity of the mixing diagrams (Figure 6) for $B_{d}$ and $B_{s}$ mesons, and their proportionality to $\left|V_{t d}\right|^{2}$ and $\left|V_{t s}\right|^{2}$ respectively, suggests immediately that the corresponding oscillation frequencies can be used as a probe of the ratio of these two CKM coefficients.

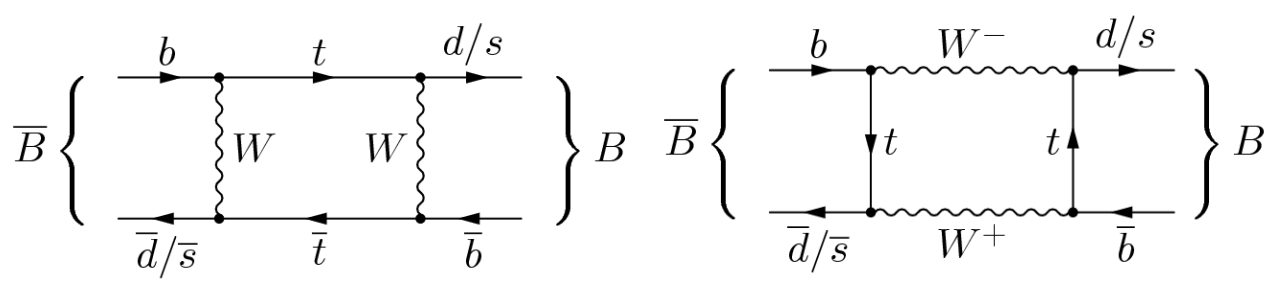

Figure 6: Standard model lowest order diagrams for $\mathrm{B}_{\mathrm{s}}$ and $\mathrm{B}_{\mathrm{d}}$ mixing.

While $\Delta m_{d}$ is extremely well known, as already discussed, the knowledge on $\Delta m_{s}$ has qualitatively improved in the last year or so. In particular, the D0 collaboration recently published [16] a double-sided limit on the Bs oscillation frequency, at 90\% CL. 
I will briefly discuss this result and then report the first direct observation of $B_{s} \bar{B}_{s}$ mixing performed by the CDF collaboration, together with the high-precision measurement of the oscillation frequency $\Delta \mathrm{m}_{\mathrm{s}}$.

\subsubsection{Introduction to $B_{s}$ mixing analyses}

The aim of these analyses is to observe an unbalance between the probability of a $B_{s}$ meson initially produced in a given flavor state to be observed decaying in the opposite state ("mixed") or in the same ("un-mixed"). The unbalance $A=\frac{N_{\text {unmix }}-N_{\text {mix }}}{N_{\text {unmix }}+N_{\text {mix }}}$ between the two probabilities can be probed as a function of proper decay time, and is predicted to be simply a cosinusoidal function of time with frequency $\frac{\Delta m_{s}}{2 \pi}$.

It is known from experiment and Standard Model predictions (see for instance the review in [3]) that $\Delta m_{s}$ is large compared to the decay frequency of $\mathrm{B}_{\mathrm{s}}$ mesons: several oscillations are expected on average before the $\mathrm{B}_{\mathrm{s}}$ actually decays.

This suggests the possibility that a Fourier transform of the time-dependent oscillation could actually be a sensitive tool for this kind of searches.

Moser and Roussarie [17] first suggested the technique widely known within the community as amplitude scan. The idea is simply to take the expected likelihood for the sample, including the oscillation signal at a given frequency $\Delta \mathrm{m}_{\mathrm{s}}$, and perform an unbinned likelihood fit to the time distribution of the events with a fixed value of $\Delta \mathrm{m}_{\mathrm{s}}$.

This yields a measured value of the oscillation amplitude A for each of these points, which is reported on a two dimensional $\mathrm{A}$ vs $\Delta \mathrm{m}_{\mathrm{s}}$ graph usually known as the sample's amplitude scan (see for example Figure 7).

An actual mixing signal will show - by

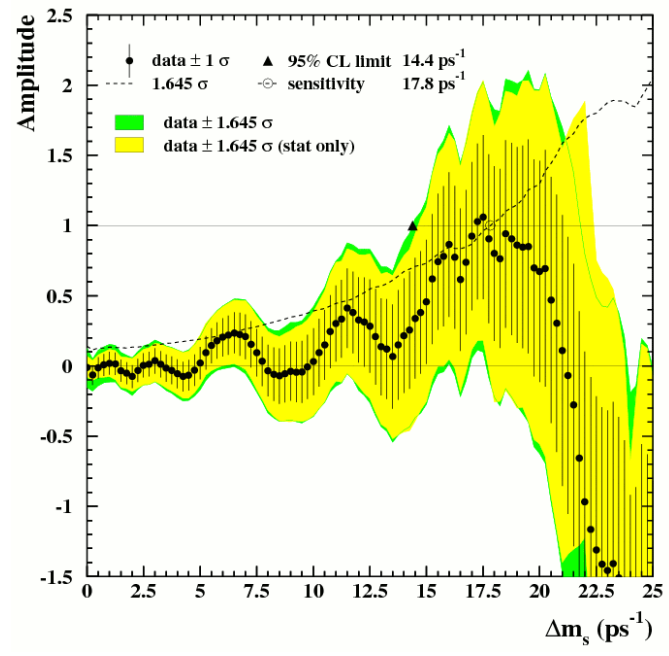

Figure 7: Example of amplitude scan taken from PDG 2004 [2]. The scan points are in black, with the relative error bars. 95\% CL bands with (green) and without (yellow) systematic uncertainties are drawn, as well as the expected average position of the top end of such bands (dashed line). construction - an amplitude on average of 1 at the right frequency, while being on average close to 0 everywhere else.

An amplitude scan like the one in Figure 7 can then be immediately used to set a lower limit on $\Delta \mathrm{m}_{\mathrm{s}}$, by simply excluding all the values for which an amplitude of 1 is not covered by a 95\% CL band around the data points. 


\subsubsection{The D0 result}

The D0 collaboration reported in [16] the result of their first analysis of 26700 $\mathrm{B}_{\mathrm{s}} \rightarrow \mathrm{D}_{\mathrm{s}}[\rightarrow \phi \pi] l v$ decays in $1 \mathrm{fb}^{-1}$ of integrated luminosity. The corresponding amplitude scan and negative log-likelihood ratio (normalized to the absolute minimum) are reported in Figure 8.
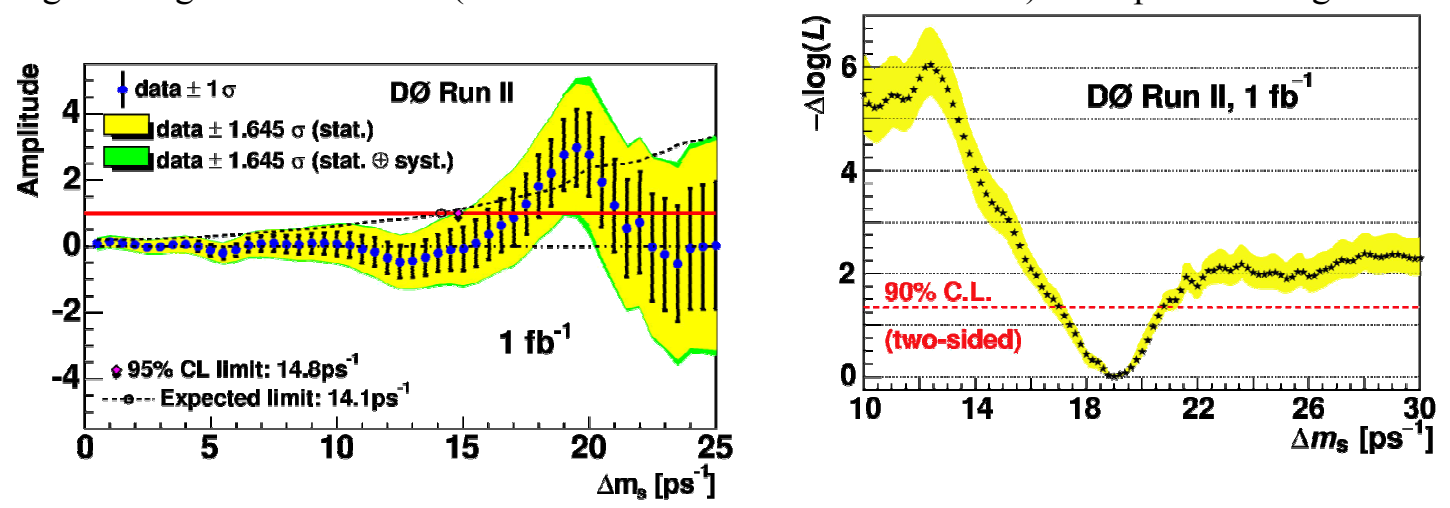

Figure 8: amplitude scan (left) and negative log-likelihood ratio for the $267001 \mathrm{D}_{\mathrm{s}}$ candidates of the $\mathrm{D} 0$ analysis.

The feature above the sensitivity threshold $\left(14.1 \mathrm{ps}^{-1}\right)$ in the amplitude scan shows a peak which is $1.6 \sigma$ from the expected signal amplitude, and $2.5 \sigma$ from 0 . The peak position corresponds to the minimum in the likelihood profile. The collaboration decides to quote a "two sided bound" corresponding to the likelihood dip: $\Delta \mathrm{m}_{\mathrm{s}} \in[17,19] \mathrm{ps}^{-1} @ 90 \% \mathrm{CL}$.

\subsubsection{The CDF observation}

The CDF collaboration has analyzed $1 \mathrm{fb}^{-1}$ of integrated luminosity, reconstructing semileptonic $\left(D_{s} l v\right)$ and fully hadronic $\left(D_{s} \pi, D_{s} \pi \pi \pi\right) B_{s}$ final states. The $D_{s}$ candidates are reconstructed in three distinct final states $\left(\phi \pi, \pi \pi \pi, \mathrm{K}^{*} \mathrm{~K}\right)$, yielding 8700 hadronic and 61500 semileptonic candidates, with signal to background ratios as high as 11.3 for the "golden" $\mathrm{B}_{\mathrm{s}} \rightarrow \mathrm{D}_{\mathrm{s}}[\rightarrow \phi \pi] \pi$ mode.

Figure 9 shows the invariant mass distribution of the $B_{s}$ candidates with $D_{s} \rightarrow \phi \pi$.

The amplitude scan and likelihood profile on the combined sample are reported in Figure 10. The black horizontal line in the likelihood scan corresponds to the $5 \sigma$ threshold. 

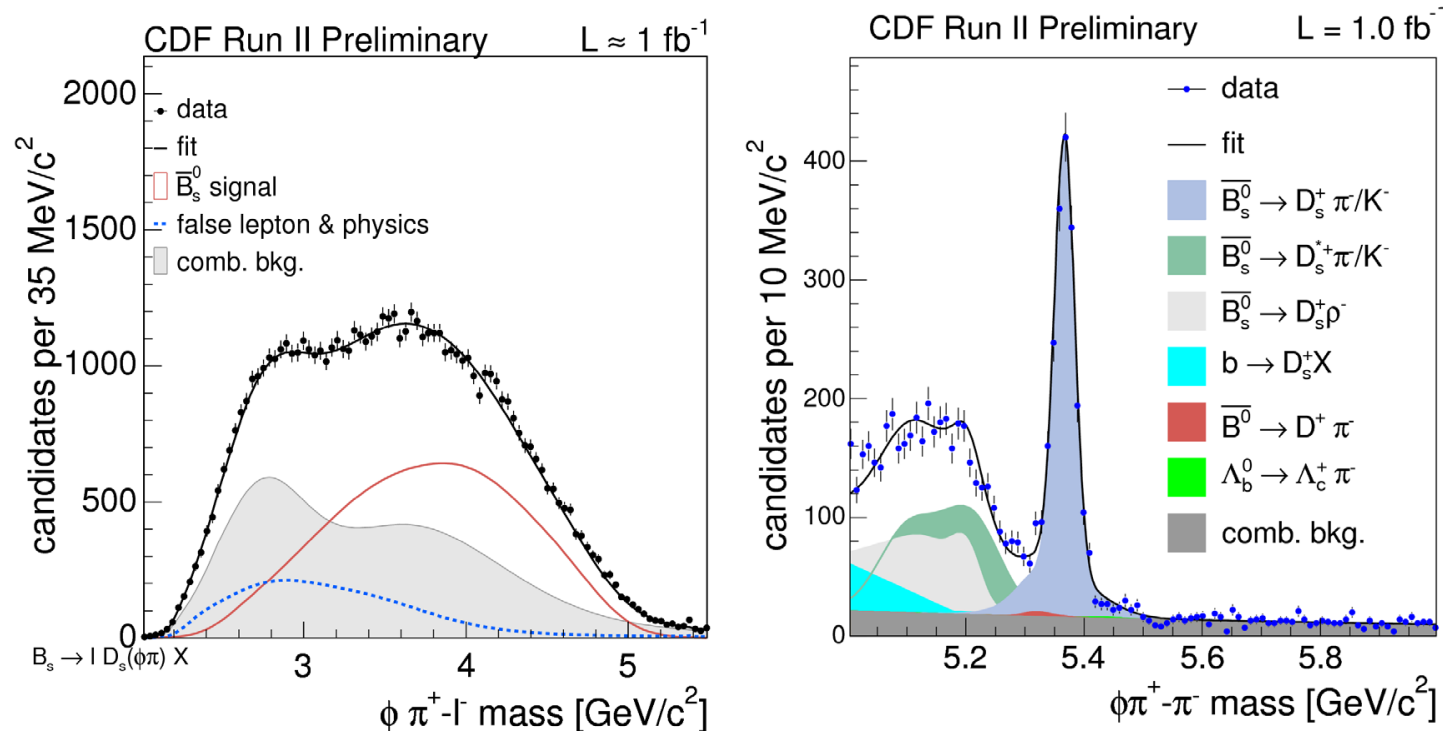

Figure 9: (Left) invariant mass distributions for the $\mathrm{lD}_{\mathrm{s}}(\rightarrow \phi \pi)$ candidates. "false lepton \& physics" refers to backgrounds from hadrons mimicking the lepton signature combined with a real $\mathrm{D}_{\mathrm{s}}$ meson, as well as physics backgrounds such as $\mathrm{B}^{0} \rightarrow \mathrm{Ds}[\rightarrow \phi \pi] \mathrm{D}^{-}[\rightarrow 1 \mathrm{X}]$.

(Right) Invariant mass distribution for $D_{s} \pi\left(D_{s} \rightarrow \phi \pi\right)$ decays, including the contributions from other Bs decay modes.
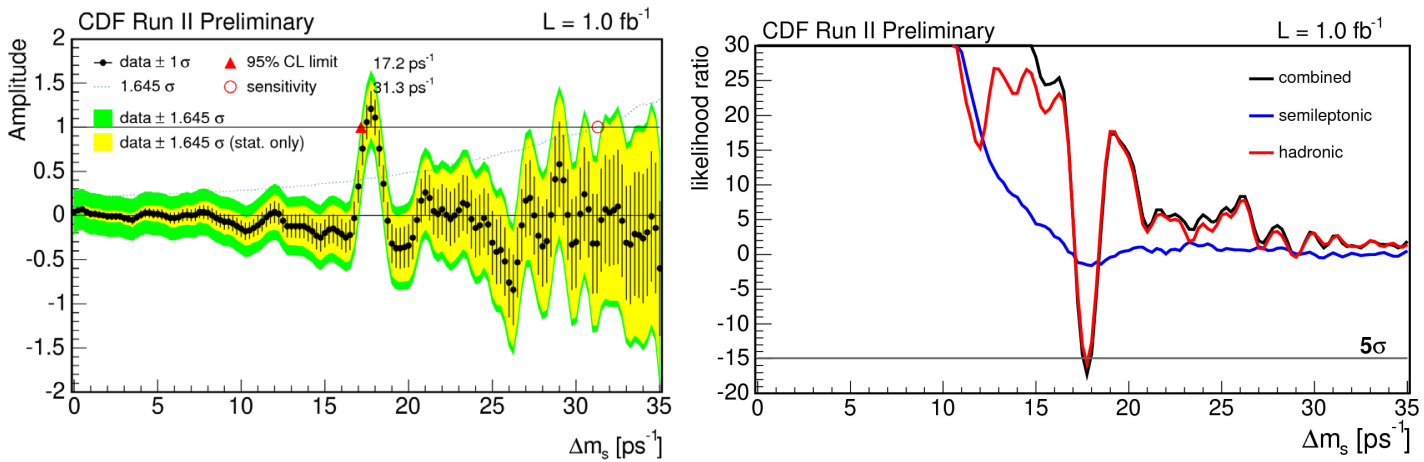

Figure 10: amplitude scan (left) and negative log-likelihood profile (right) for the combination of all the samples reconstructed in the CDF analysis. The red and blue lines in the likelihood scan correspond to the hadronic and semileptonic sub-samples taken individually.

The probability that random fluctuations could produce a signal comparable to what seen in Figure 10 is $8 \times 10^{-8}$, which exceeds $5 \sigma$ significance. The resulting measurements of $\Delta m_{s}$ and $\left|\frac{V_{t d}}{V_{t s}}\right|$ are:

$$
\begin{aligned}
& \Delta m_{s}=17.77 \pm 0.10(\text { stat } .) \pm 0.07(\text { syst. }) \\
& \left|\frac{V_{t d}}{V_{t s}}\right|=0.2060 \pm 0.0007(\exp )_{-0.0060}^{+0.0081}(\text { theo })
\end{aligned}
$$


For the derivation of the second of these measurements, the combination of experimental results on $\Delta m_{d}$ found in PDG 2006 [3] and the LQCD coefficient provided in [19] were used. The latter is to date the dominant source of uncertainty on this determination of $\left|\frac{V_{t d}}{V_{t s}}\right|$.

\section{Conclusions}

The lattice community is contributing to the advancement of our knowledge of the Standard Model of elementary particles through the determination of numbers which are often the sole link between the experimental side of heavy flavor physics and the underlying basic Standard Model quantities. Experiments are becoming increasingly dependent on LQCD predictions in order to constrain and over-constrain the theoretical framework and find indications of new effects, and therefore more and more demanding.

Currently the precision on some of these constraints, like that on $\left|V_{t d} / V_{t s}\right|$, is systematically limited by LQCD uncertainties and could greatly benefit from more accurate determinations in order to fully exploit the precision of the experimental result, as well as crosschecks against other derivations when possible (see for instance [20]).

\section{Acknowledgment}

I would like to thank the organizers of the conference for giving me the opportunity of a full immersion into the LQCD community. I would like to thank also Elisabetta Barberio, Michael Creutz, Riccardo Faccini, Hung-Chung Fang, Andreas Kronfeld, Zoltan Ligeti, Michele Papucci, Kevin Pitts, Anders Ryd, Yoshihide Sakai and Marjorie Shapiro for their precious input at various stages in the preparation of this review.

\section{References}

[1] CKMfitter Group (J. Charles et al.) Eur. Phys. J. C41, 1-131 (2005) [hep-ph/0406184] and http://ckmfitter.in2p3.fr

[2] S. Eidelman et al., Phys. Lett B592, 1 (2004)

[3] W.-M. Yao et al., J. Phys. G 33, 1 (2006)

[4] CLEO collaboration, M. Artuso et al., Phys. Rev. Lett. 95, 251801 (2005)

[5] FERMILAB/MILC/HPQCD collaborations, Phys. Rev. Lett. 94, 011601 (2005) [hep-ph/0408306]

[6] BABAR collaboration, hep-ex/0605030 and CLEO collaboration, contributed paper at the $33^{\text {rd }}$ International Conference on High Energy Physics, July 26-August 2, 2006, Moscow (ICHEP06)

[7] BELLE collaboration, hep-ex/060408 and contribution to the $33^{\text {rd }}$ International Conference on High Energy Physics, July 26-August 2, 2006, Moscow (ICHEP06)

[8] Heavy Flavor Averaging Group E. Barberio et al., hep-ex/0603003

[9] HPQCD, Phys. Rev. Lett. 95, 212001 (2005) 
[10]BABAR collaboration hep-ex/0607077, contributed paper at the $33^{\text {rd }}$ International Conference on High Energy Physics, July 26-August 2, 2006, Moscow (ICHEP06)

[11] FOCUS collaboration, Phys. Lett. B607, 233-242 (2005) [hep-ex/0410037]

[12] BELLE collaboration [hep-ex/0510003]

[13] CLEO collaboration, contributed paper at the $33^{\text {rd }}$ International Conference on High Energy Physics, July 26-August 2, 2006, Moscow (ICHEP06)

[14] Koerner and Schuler, Z. Phys. C38, (1988)

[15] BELLE collaboration [hep-ex/0506079]

[16] D0 collaboration, Phys. Rev. Lett. 97, 021802 (2006)

[17] H.G. Moser and A. Roussarie, Nucl. Instrum. Methods Phys. Res. Sect. A384, 491 (1997)

[18] CDF collaboration [hep-ex/060904], submitted to Phys. Rev. Lett.

[19] M. Okamoto, Proc. Sci. LAT2005 (2005) 013 [hep-lat/0510113]

[20] B. Grinstein et al., Nucl. Phys. B380, 369-376 (1992) [hep-ph/9204027] 